\title{
Constraints on UHECR sources and their environments, from fitting UHECR spectrum and composition, and neutrinos and gammas
}

\author{
Marco Stein Muzio* \\ Center for Cosmology and Particle Physics, \\ Department of Physics, New York University, 726 Broadway, New York, New York, USA \\ E-mail: msm659@nyu. edu \\ Michael Unger \\ Institut für Kernphysik, \\ Karlsruher Institut für Technologie, 76021 Karlsruhe, Germany \\ E-mail: Michael. Unger@kit.edu

\section{Glennys R. Farrar} \\ Center for Cosmology and Particle Physics, \\ Department of Physics, New York University, 726 Broadway, New York, New York, USA \\ E-mail: gf25 @ nyu. edu
}

\begin{abstract}
We perform high-precision joint fitting of the ensemble of Auger extragalactic spectrum, composition, IceCube neutrino spectrum and upper limits, and Fermi-LAT diffuse gamma spectrum, to constrain the properties of UHECR sources, the source environments, source evolution and distance to nearest source. At present, the largest source of uncertainty comes from the LHC-tuned hadronic event generators, limiting the ability to derive decisive conclusions on all of the above topics. Nonetheless, interesting constraints on UHECR, neutrino and gamma ray sources can (and will) be presented.
\end{abstract}

36th International Cosmic Ray Conference -ICRC2019-

July 24th - August 1st, 2019

Madison, WI, U.S.A.

\footnotetext{
* Speaker.
} 


\section{Introduction}

The origin of ultrahigh energy cosmic rays (UHECRs), cosmic rays with energies in excess of $1 \mathrm{EeV}\left(10^{18} \mathrm{eV}\right)$, has long been an open question in astroparticle physics. Flux and composition measurements of UHECRs by the Pierre Auger Observatory (Auger) and the Telescope Array (TA) have provided a much clearer picture of the flux arriving at Earth. However, these observables are an amalgamation of UHECRs accelerated by a variety of astrophysical sources over cosmic time and processed by interactions, both in their source environments and in propagation to Earth. To overcome this, we study the properties of typical sources indirectly by employing phenomenological source models that are agnostic about the specific astrophysical system accelerating CRs to ultrahigh energies. For this purpose, we use the Unger-Farrar-Anchordoqui (UFA) source model [1]. UFA has been shown to give high-precision fits to both the Auger spectrum and composition data by taking into account photodisintegration interactions in the source environment. The model characterizes the source using a number of physical parameters which can be tuned to obtain a good fit to data. These best-fit parameters give insight into the physical characteristics typical sources can have compatible with observations. In this way, constraints can be placed on UHECR sources.

Other observables also carry information about the properties of UHECR sources, however. Neutrino and gamma-ray secondaries are produced by CR interactions inside their source environment and in propagation to Earth. These secondaries act as neutral messengers probing both the source environment and global properties of UHECR sources. Auger [2] and IceCube [3] provide the strongest upper-bounds on the neutrino flux between $\sim 3 \mathrm{PeV}$ and $\sim \mathrm{EeV}$. To complement these bounds, the Fermi-Large Area Telescope (LAT) gives an upper-bound on the diffuse gamma-ray flux in the $100 \mathrm{MeV}$ to $\sim \mathrm{TeV}$ energy range $[4,5]$. By calculating the predicted neutrino and gamma-ray fluxes in UFA models we can further exclude models which violate these bounds.

These multimessenger studies (in particular the neutrino flux limits) lead to constraints on the characteristic energy of the photon field surrounding the source. The UHECR spectrum and composition proves to be the strongest constraint on the source evolution, with a preference for a star-formation-like source evolution, and we report on constraints on the distance to the nearest source. Finally, we consider the possibility of two distinct primary CR populations, as could arise, e.g., if there are different source types. In particular, we explore a secondary pure-proton component and find that, for both hadronic interaction models considered, this improves the fit to the UHECR data - by up to $5 \sigma$ — without producing a conflict with gamma-ray and neutrino constraints.

\section{Results}

A summary of our results, as was presented at the conference, follows below. For a detailed discussion of this work see [6].

To study the effects of source evolution we considered two source evolution parametrizations: a star-formation rate (SFR) evolution [7],

$$
\xi(z) \propto \frac{(1+z)^{a}}{1+[(1+z) / b]^{c}},
$$


where $a=3.26 \pm 0.21, b=2.59 \pm 0.14$, and $c=5.68 \pm 0.19$; and a single power-law evolution with an exponential cutoff,

$$
\xi(z)= \begin{cases}(1+z)^{m} & z<z_{0} \\ \left(1+z_{0}\right)^{m} e^{-\left(z-z_{0}\right)} & z \geq z_{0},\end{cases}
$$

where in both cases $\xi(z)$ is the comoving power density of CR sources at redshift $z$ relative to its value today. After marginalizing over all other source parameters, we found the best-fit models as a function of source evolution for UFA sources (see Fig. 1 for an example of a typical fit).

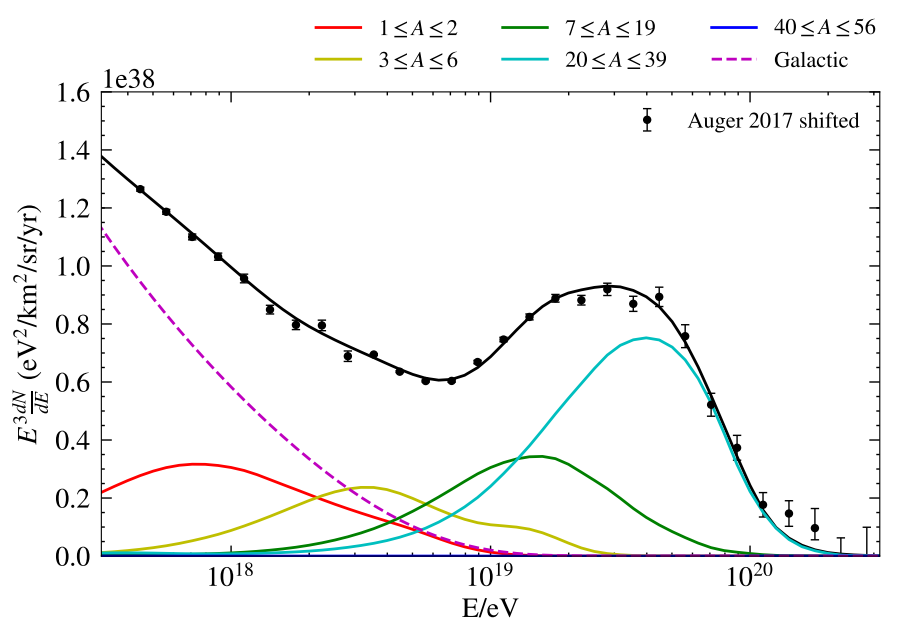

(a)

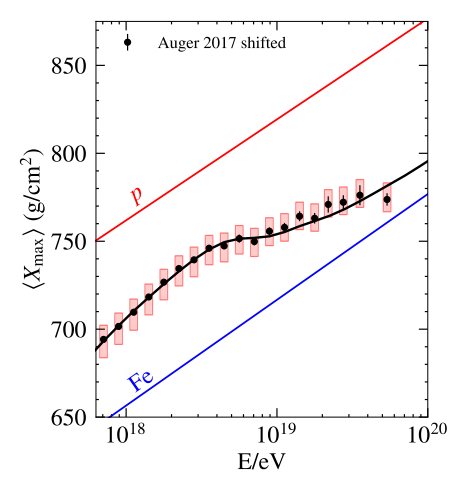

(b)

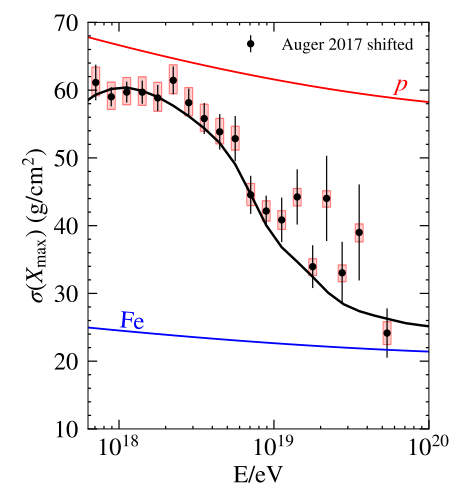

(c)

Figure 1: Best-fit CR spectrum (top panel) and composition (bottom panels) for a SFR evolution using a blackbody photon field and hadronic interaction model SIBYLL2.3C. Top: CR spectrum (black line) along with the Auger 2017 spectrum (black circles). For illustration, the spectrum is also shown broken down by mass group (colored lines). Bottom: CR composition-sensitive observables $X_{\max }$ (left) and $\sigma\left(X_{\max }\right)$ (right) plotted along with their Auger 2017 values (black circles), where error bars and shaded boxes denote statistical and systematic uncertainties. SIB YLL2.3C predictions for pure-proton (red) and pure-iron (blue) compositions are also shown.

The resulting best-fit reduced $\chi^{2}$ values are shown in Fig. 2 as a function of source evolution for several photon field parametrizations and two post-LHC hadronic interaction models, EPOSLHC [8] and SibYLL2.3C [9]. Regardless of the photon field parametrization or the hadronic 
interaction model assumed the conclusion is the same: positive source evolutions, like SFR, for which there was more CR production in the past, are able to better describe the Auger spectrum and composition data.

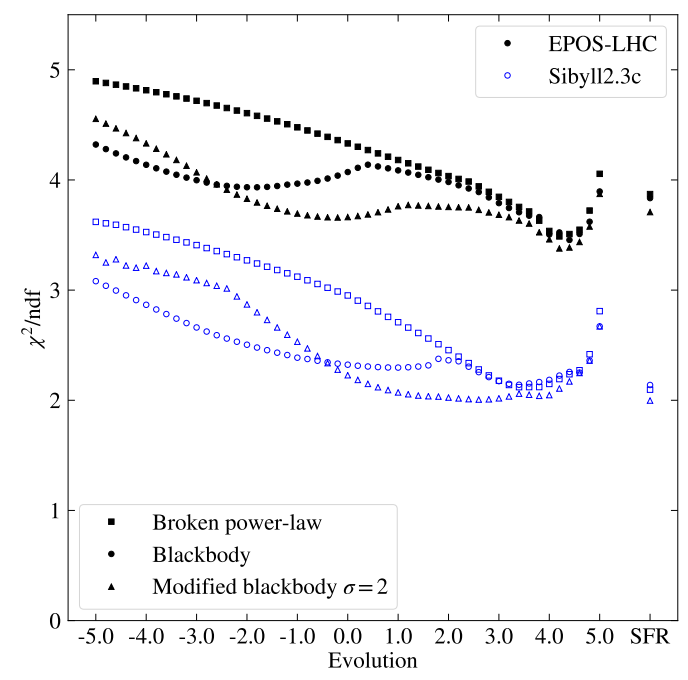

Figure 2: Reduced $\chi^{2}$ of best-fit models as a function of source evolution index $m$. Results are shown for several photon field parametrizations (symbols) and hadronic interaction models (colors).

While gamma-ray data does not currently constrain source evolutions in the range $-5 \leq m \leq$ +5 which we considered, there is a clear evolution-dependent shape to the gamma-ray flux, as is shown in Fig. 3. Similarly, current neutrino bounds do not constrain the CR source evolution and current neutrino experiments will require at least another decade of exposure before they will be able to do so [6].

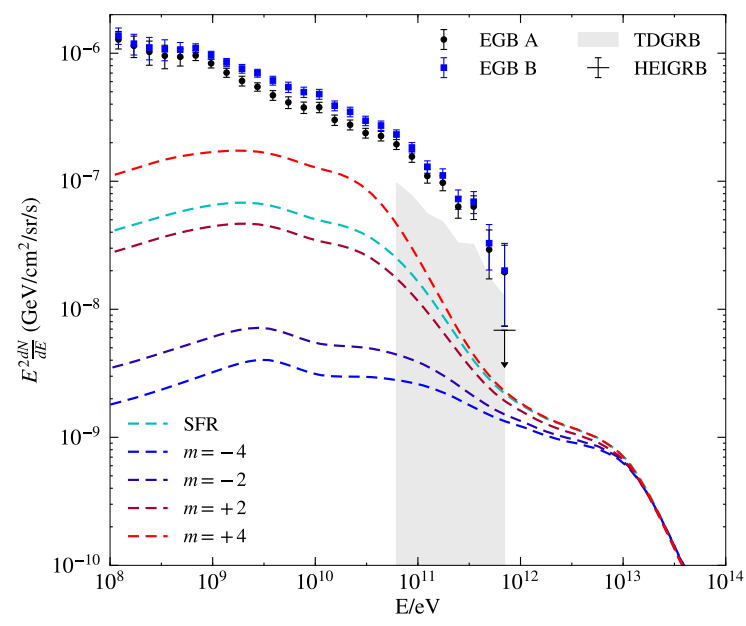

Figure 3: Gamma-ray fluxes for various $\mathrm{CR}$ source evolutions (dashed colored lines). The extragalactic gamma-ray background (EGB) flux for galactic foreground model A (black circles) and model B (blue squares), upper-bound on the truly diffuse gamma-ray background (TDGRB, grey shaded region), and upperbound on the isotropic gamma-ray background (IGRB) flux in the highest energy bin (black upper-bound) are also plotted. 
Current neutrino bounds are constraining, however, for the temperature (or peak photon energy) of the photon field in the source environment. As the temperature of the source photon field increases, the threshold energy for photopion production decreases, and the number of neutrinos produced increases (as is illustrated in Fig. 4). This allows us to constrain the blackbody temperature of the source to be less than $4000 \mathrm{~K}$, or to have a peak photon energy of less than $500 \mathrm{meV}$, based on current neutrino bounds.

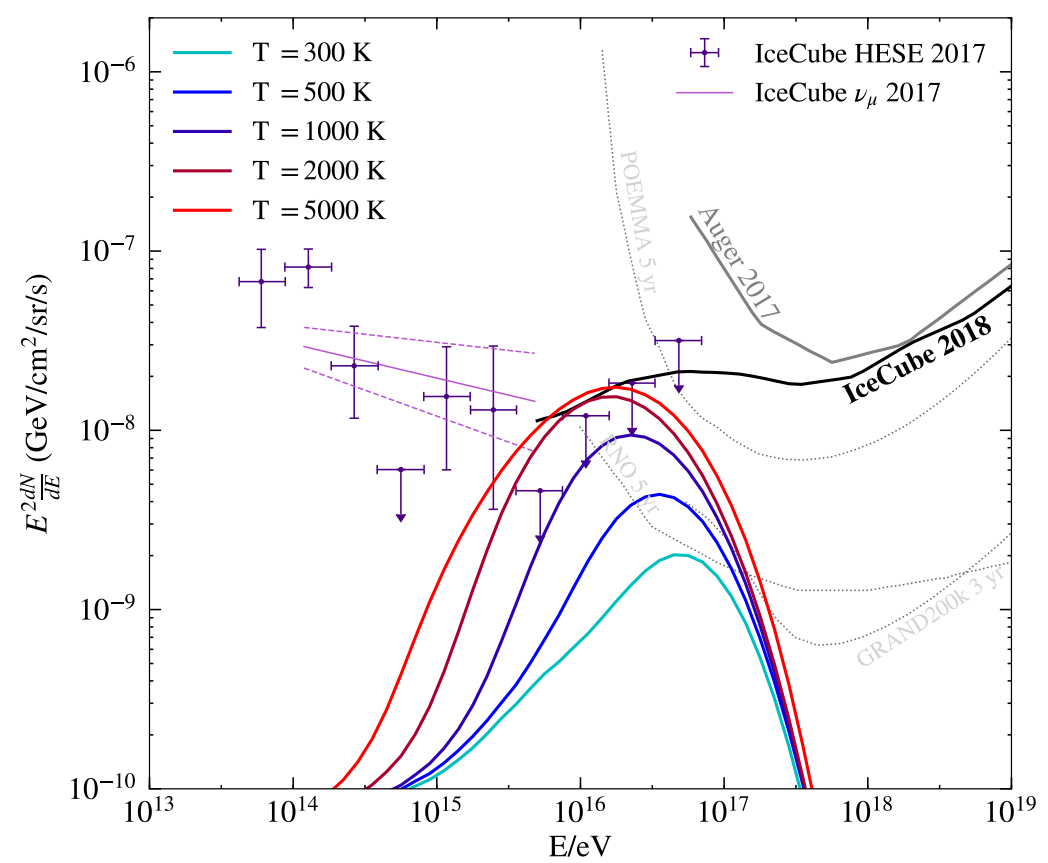

Figure 4: Neutrino flux for various blackbody source photon field temperatures (colored lines). Auger 2017 (grey line) and IceCube 2018 (black line) upper-bounds on the cosmic neutrino flux are shown, along with the astrophysical neutrino flux measured by IceCube (purple data points and inferred spectrum). The predicted sensitivites of several future neutrino detectors, POEMMA [10], RNO [11], and GRAND [12], are also shown (grey dotted lines).

Throughout our study, we considered two classes of CR composition injected into the source environment: a composition with a narrow mass range, approximated by a single mass injection, and an extended, Milky Way-like composition. While the single-mass composition describes the spectral data well with a silicon-like mass injection, the Milky Way-like (or galactic mix) composition produces a predicted spectrum which overshoots observations around the cutoff. This overshoot is due to the fact that the galactic mix includes heavy nuclei like iron. While this could be interpreted as indicating that a narrow mass range better represents the CR composition injected into UHECR source environments, it is important to note that the behaviour of the spectrum in the cutoff region is very sensitive to the distance to the nearest source of UHECRs. To study if a non-zero distance to the nearest source could improve the fits where a galactic mix composition is used, we conducted fits using a source evolution with no sources up to comoving distance $D_{\text {min }}$ and a SFR evolution beyond $D_{\min }$. By using a non-zero nearest source distance, the spectral cutoff in models using a galactic mix injection is able to describe the data to much higher accuracy, as is illustrated in Fig. 5. Moreover, this allows us to place a constraint on the distance to the nearest 
source, under the assumption of a given injected composition. For galactic mix injections, the nearest source (in the Auger field of view) is likely to be $30-50 \mathrm{Mpc}$ from Earth, while for single-mass injections it is likely to be within $\sim 5 \mathrm{Mpc}$ from Earth.

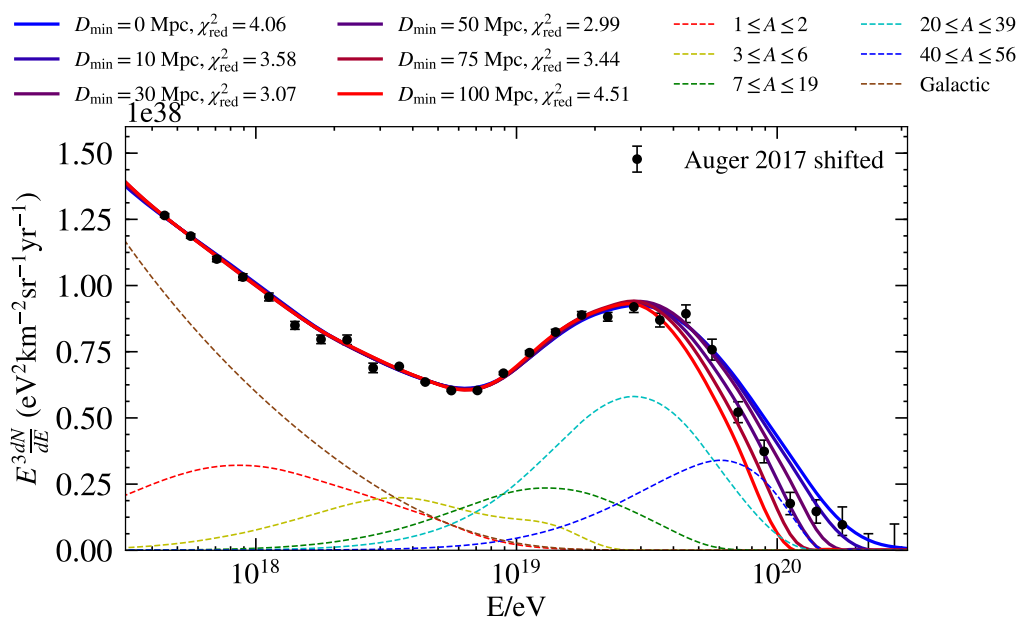

Figure 5: Effect of the distance to the nearest source on the best-fit CR spectrum for models using a Milky Way-like composition for $\mathrm{CR}$ injection into the source environment. For illustration, the spectrum is broken down by mass group (dashed colored lines) for the $D_{\min }=50 \mathrm{Mpc}$ case.

Finally, we consider the possibility that there exists a secondary and sub-dominant pure-proton component to the spectrum. Such a component helps to improve the fit on two fronts: it lightens and variegates the too-heavy and too-pure composition generically produced by photodisintegration models at energies above the ankle. To model this secondary component we parametrized the proton spectrum escaping the source by

$$
\phi_{p} \sim f_{p} E^{-1} e^{-E / E_{\max }^{\mathrm{UHE} p}}
$$

where $E_{\max }^{\mathrm{UHE} p}$ is the cutoff energy and $f_{p}$ is the normalization given by

$$
f_{p}=\frac{\int_{E_{\mathrm{ref}}}^{\infty} E \phi_{p} \mathrm{~d} E}{\int_{E_{\mathrm{ref}}}^{\infty} E\left(\phi_{p}+\phi_{\mathrm{UFA}}\right) \mathrm{d} E}
$$

where $E_{\mathrm{ref}}=10^{19} \mathrm{eV}$ and $\phi_{\mathrm{UFA}}$ is the escaping flux of the primary UFA component. As shown in Fig. 6, this secondary component generically improves the fit over a broad range of values for $f_{p}$ and $E_{\max }^{\mathrm{UHE} p}$. Depending on the hadronic interaction model assumed, this improvement can be as much as $5 \sigma$ over the best-fit model without a secondary component.

Importantly, the addition of this secondary pure-proton component is unconstrained by current gamma-ray and neutrino bounds. However, future neutrino detectors, like RNO and GRAND, will be able to detect or constrain such a component, as can be seen in Fig. 7. Moreover, the addition of this secondary component hints at the possibility of a population of CRs at the highest energies with rigidities above $10 \mathrm{EV}$ (as is illustrated in Fig. 8 for the maximum proton signal compatible with data). If such a population exists, their deflections in the galactic magnetic field would be much smaller than for other, lower rigidity CRs, allowing for the possibility of CR astronomy. 


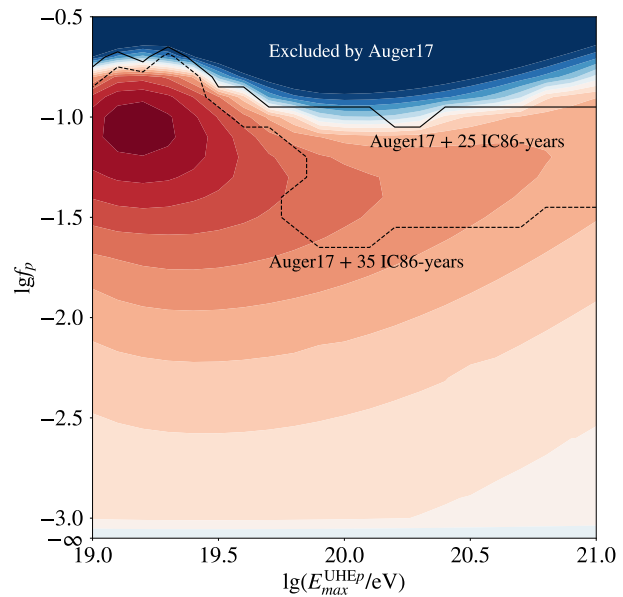

(a)

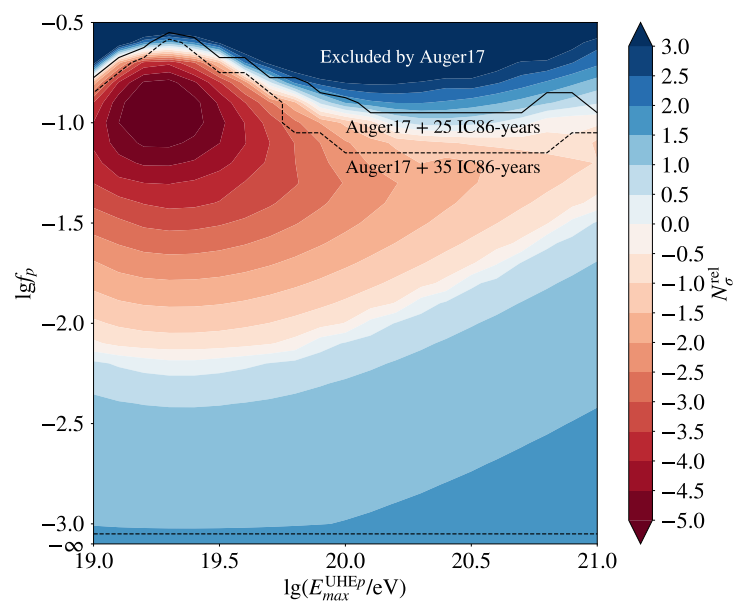

(c)

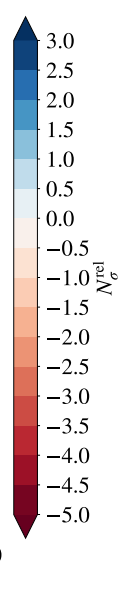

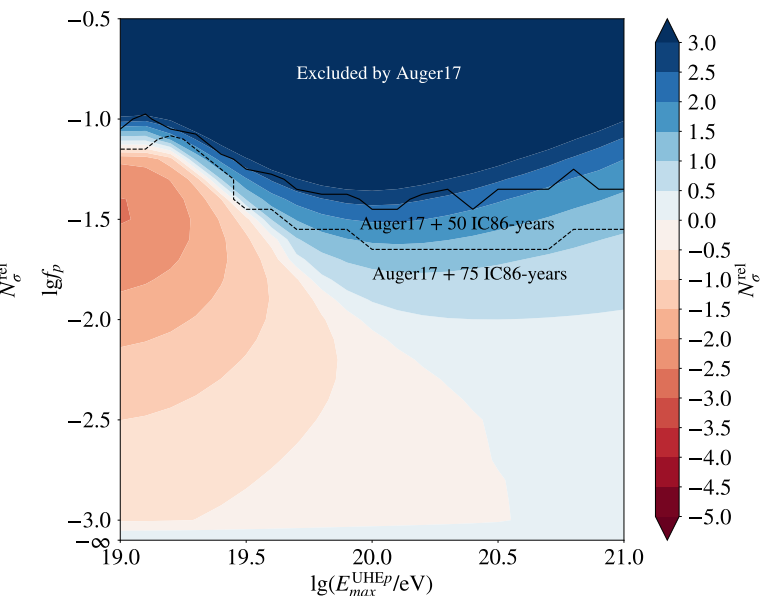

(b)

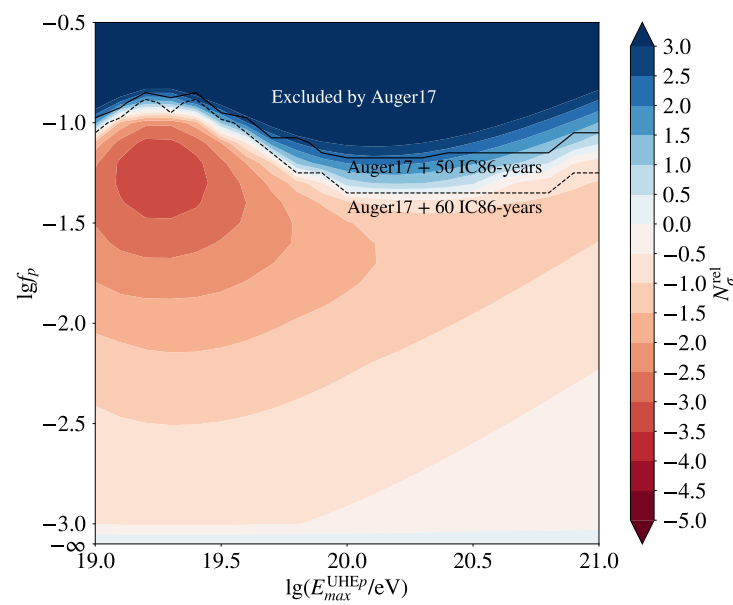

(d)

Figure 6: Effect on fit due to an additional pure-proton component with (a) single-mass EPOS-LHC, (b) single-mass SibYLL2.3C, (c) galactic mix EPOS-LHC, and (d) galactic mix SiBYLL2.3C mixed composition models. The color indicates the number of standard deviations, $N_{\sigma}^{\text {rel }}$, from the corresponding best-fit UFA15 model without the extra component. Black contours indicate the combined CR-neutrino bounds with the dark blue region excluded by current Auger data at the 90\% CL.

Current neutrino and cosmic ray data is compatible with more than $10 \%$ of the CRs above $50 \mathrm{EeV}$ being protons, regardless of the assumed hadronic interaction model or composition at injection. Future mass-senstive UHECR detectors, such as AugerPrime [13] and POEMMA, will be able to constrain this possibility.

\section{Conclusion}

We have shown that applying a multimessenger approach to UHECR source models can be very effective at illuminating the types of source properties that can be describe observations. Applying a realistic, but astrophysical source-agnostic, UHECR source model allows for strong inferences to be made from the Auger spectrum and composition data. In particular, we find that 
positive and SFR-like source evolutions are strongly favored by current observations. Further, these evolutions are not yet constrained by either gamma-ray or neutrino bounds.

Neutrinos can, however, be an effective probes of properties inside the source environment such as the blackbody temperature of the ambient photon field surrounding the source. Current neutrino bounds constrain this temperature to be less than $4000 \mathrm{~K}$, or less than $500 \mathrm{meV}$ for the peak photon energy of the photon field.

The cutoff of the CR spectrum has been shown to be an effective probe of the nearest source distance, under the assumption of a given injected composition into the source environment. We find that this favors the nearest source (in the Auger field of view) to be $30-50 \mathrm{Mpc}$ from Earth if the injected composition is Milky Way-like and within $\sim 5 \mathrm{Mpc}$ of Earth if the injected composition is relatively pure.

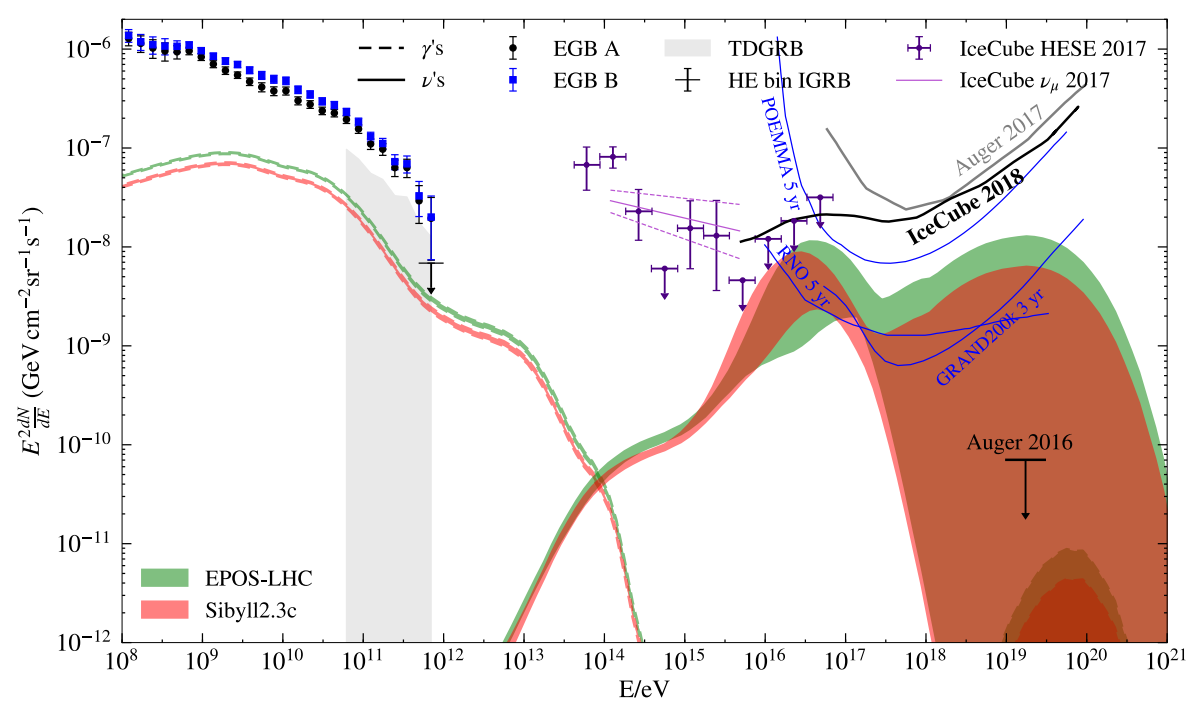

Figure 7: Range of gamma-ray (dashed lines) and neutrino (solid lines) fluxes produced by best-fit models in the $f_{p}-E_{\max }^{\mathrm{UHE} p}$ parameter space compatible with current $\mathrm{CR}$ and neutrino data, injecting either a single-mass or galactic mix composition into the source environment. Results are shown for both EPOS-LHC (green) and SIBYLL2.3C (red) hadronic interaction models. Predicted sensitivities of future neutrino detectors are also shown (blue lines). The Auger 2016 upper-bound ([14], black) on UHE gamma-rays is also shown.

Finally, we find that the data strongly favors the addition of a secondary, sub-dominant pureproton component to the spectrum. This extra component is both unconstrained and improves the model's description of the data over a broad range of parameter values. Moreover, such a component hints at the possibility of a population of CRs with rigidites above $10 \mathrm{EV}$ at the highest energies, with more than $10 \%$ of the CRs above $50 \mathrm{EeV}$ allowed to be protons while still being compatible with current data, regardless of the hadronic interaction model assumed. Future mass-sensitive UHECR detectors, such as AugerPrime and POEMMA, as well as future neutrino detectors, such as RNO and GRAND, could constrain or even detect the existence of this secondary component of the CR spectrum. 


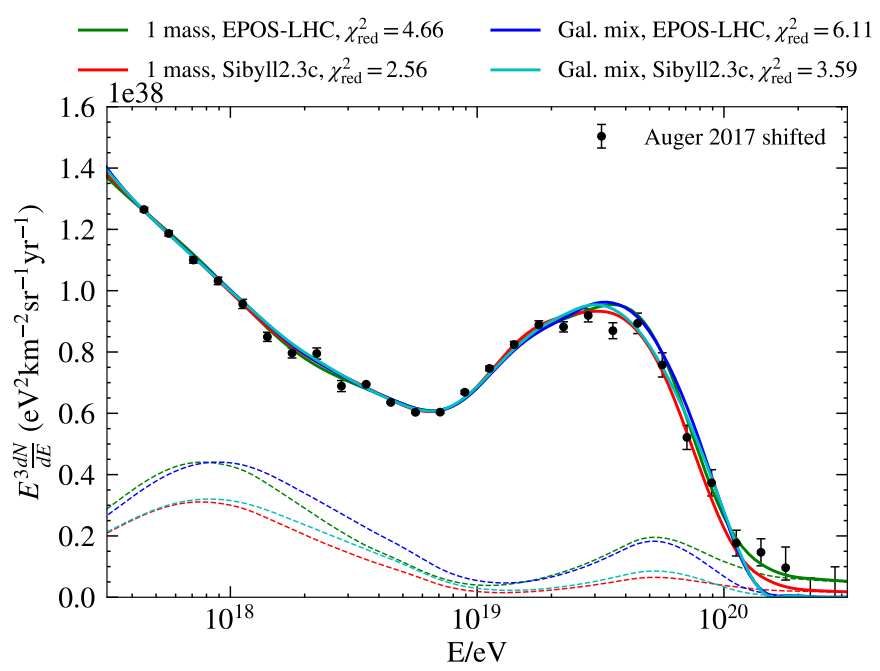

Figure 8: Predicted CR spectra (solid lines) in two-component models producing the maximum proton signal at Earth above $50 \mathrm{EeV}$ compatible with current data. For illustration, the proton component of the spectrum is also shown (dashed lines).

\section{References}

[1] M. Unger, G. R. Farrar, and L. A. Anchordoqui, Origin of the ankle in the ultrahigh energy cosmic ray spectrum, and of the extragalactic protons below it, Phys. Rev. D 92, 123001 (2015), arXiv:1505.02153.

[2] E. Zas, Searches for neutrino fluxes in the EeV regime with the Pierre Auger Observatory, in proceedings of 35th International Cosmic Ray Conference (ICRC 2017), POS ( ICRC2017) 972 (2017).

[3] M. G. Aartsen et al. (IceCube), Differential limit on the extremely-high-energy cosmic neutrino flux in the presence of astrophysical background from nine years of IceCube data, Phys. Rev. D 98, 062003 (2018), arXiv:1807.01820.

[4] M. Ackermann et al. (Fermi-LAT), The spectrum of isotropic diffuse gamma-ray emission between $100 \mathrm{MeV}$ and $820 \mathrm{GeV}$, Astrophys. J. 799, 86 (2015), arXiv:1410.3696.

[5] M. Ackermann et al. (Fermi-LAT), Resolving the Extragalactic $\gamma$-Ray Background above 50 GeV with the Fermi Large Area Telescope, Phys. Rev. Lett. 116, 151105 (2016), arXiv:1511.00693.

[6] M. S. Muzio, M. Unger, and G. R. Farrar, Progress towards characterizing ultrahigh energy cosmic ray sources, arXiv:1906.06233.

[7] B. Robertson et al., Cosmic reionization and early star-forming galaxies: A joint analysis of new constraints from Planck and the Hubble Space Telescope, Astrophys. J. 802, 19 (2015), arXiv:1502.02024.

[8] T. Pierog et al., EPOS LHC: Test of collective hadronization with data measured at the CERN Large Hadron Collider, Phys. Rev. C 92, 034906 (2015), arXiv:1306.0121.

[9] F. Riehn et al., A new version of the event generator Sibyll, in proceedings of 34th International Cosmic Ray Conference (ICRC 2015): The Hague, The Netherlands, July 30-August 6, 2015, POS ( ICRC2015) 558 (2016), arXiv:1510.00568. 
[10] J. Krizmanic, POEMMA: Probe Of Extreme Multi-Messenger Astrophysics, in proceedings of Ultra High Energy Cosmic Rays (UHECR 2018): Paris, France, October 8-12, 2018, EPJ Web Conf. 210 06008 (2019).

[11] A. L. Connolly, The Radio Neutrino Observatory, talk given at the 2019 APS April Meeting, Denver, Colorado, USA, April 13-16, 2019, http://meetings.aps.org/Meeting/APR19/Session/R08.3.

[12] J. Alvarez-Muñiz et al. (GRAND), The Giant Radio Array for Neutrino Detection (GRAND): Science and Design, arXiv:1810.09994.

[13] A. Aab et al. (Pierre Auger), The Pierre Auger Observatory Upgrade - Preliminary Design Report, arXiv:1604.03637.

[14] A. Aab et al. (Pierre Auger), Search for photons with energies above $10^{18} \mathrm{eV}$ using the hybrid detector of the Pierre Auger Observatory, JCAP 1704 (2017), arXiv:1612.01517. 